Results: The study cohort included 192.037 patients with gout, $82.6 \%$ of those were males. There was a progressive increase in the number of hospitalised patients with gout from 12851 patients in 2005 to 23318 in 2015; this was associated with an increase in mortality, reaching its highest value in 2015 with a $4.9 \%$ of gout hospitalised patients. The average age at dead in 2015 was 79.2 years and 85.16 years in male and female respectively, an age slightly lower than in the general population. The average cost in these hospitalised patients was $4931 €$, reaching a peak of $5384 €$ in the last year. The hospital stay reached its lowest numbers in 2015 with an average of 8.9 days per patient. These comorbidities had statistical association with an added mortality risk in cerebrovascular disease (odds ratio [OR] 1.57, 95\% confidence interval [Cl]1.46-1.49), liver disease (OR $2.6195 \% \mathrm{Cl} 2.34-2.9$ ), kidney disease (OR $1.3495 \% \mathrm{Cl} 1.28-1.41$ ), dementia (OR $2.1395 \% \mathrm{Cl} 1.88-2.42$ ). On the contrary, in type 2 diabetes (OR 0.92 $95 \% \mathrm{Cl} 0.87-0.96$ ), we found a statistically significant lower mortality risk. Furthermore, it was found a statistically reduced mortality risk in females (OR 0.85 $95 \% \mathrm{Cl} 0.8-0.9)$

Abstract FRI0242 - Table 1. Incidence, mortality, stays and annual costs of hospitalisation for gout of the Health National System

\begin{tabular}{lcccc}
\hline Year & $\begin{array}{c}\text { Number of } \\
\text { patients }\end{array}$ & $\begin{array}{c}\text { Mortality } \\
\text { Number } \\
(\%)\end{array}$ & $\begin{array}{c}\text { Average hospital stay (SD)/cost } \\
\text { (SD) }\end{array}$ & $\begin{array}{c}\text { Cost/year } \\
\text { In millions } \\
€\end{array}$ \\
\hline $\mathbf{2 0 0 5}$ & 12850 & $532(4.1 \%)$ & $11.00 / 4219$ & 54.2 \\
$\mathbf{2 0 0 6}$ & 13163 & $479(3.6 \%)$ & $10.63 / 4269$ & 56.2 \\
$\mathbf{2 0 0 7}$ & 13896 & $544(3.9 \%)$ & $10.46 / 4459$ & 61.6 \\
$\mathbf{2 0 0 8}$ & 15292 & $601(3.9 \%)$ & 10.584838 & 74 \\
$\mathbf{2 0 0 9}$ & 16172 & $630(3.9 \%)$ & $10.33 / 4997$ & 80.8 \\
$\mathbf{2 0 1 0}$ & 16803 & $658(3.9 \%)$ & $9.96 / 5205$ & 87.5 \\
$\mathbf{2 0 1 1}$ & 18482 & $781(4.2 \%)$ & $9.62 / 5385$ & 99.5 \\
$\mathbf{2 0 1 2}$ & 19179 & $897(4.7 \%)$ & $9.21 / 5247$ & 100.6 \\
$\mathbf{2 0 1 3}$ & 20.777 & $926(4.5 \%)$ & $9,09 / 5166$ & 107.3 \\
$\mathbf{2 0 1 4}$ & 22.105 & $991(4.5 \%)$ & $8,94 / 5080$ & 112.3 \\
$\mathbf{2 0 1 5}$ & 23.318 & 1139 & $8,90 / 5384$ & 125.5 \\
& & $(4.9 \%)$ & & 959.5 \\
Total & 192.037 & 8178 & $9,74 / 4999$ & \\
& & $(4.3 \%)$ & &
\end{tabular}

Conclusions: In Spain we have a progressive increase in the hospital admissions for gout, higher mortality rates and higher healthcare costs. This shows the need for changes in prevention and management of gout disease.

Disclosure of Interest: None declared

DOI: 10.1136/annrheumdis-2018-eular.7554

\section{FRI0243 ANAKINRA FOR CALCIUM PYROPHOSPHATE CRYSTAL ARTHRITIS: AN EFFICIENT, SAFE ALTERNATIVE TREATMENT}

M. Thomas, M. FORIEN, E. PALAZZO, P. DIEUDÉ, S. OTTAVIANI.

Rheumatology, Hôpital Bichat, Paris, France

Background: Calcium pyrophosphate (CPP) deposition is a frequent joint disease with increased prevalence in older people in whom treatment of acute CPP arthritis with conventional therapies such as colchicine, corticosteroids or nonsteroidal anti-inflammatory drugs (NSAIDs) could be contraindicated or not used at an optimal dose. As recommended in gout, anakinra might represent an alternative treatment for acute arthritis. In contrast, the relevance of anakinra in acute CPP arthritis has not given much attention.

Objectives: We further aimed to analyse the efficacy and safety of anakinra in acute CPP arthritis in a large cohort.

Methods: We retrospectively included all patients receiving anakinra for acute CPP arthritis between January 2011 and 2017. Medical history data were collected including hypertension, diabetes mellitus, cardiovascular disease, history of gastroduodenal ulcer, renal impairment and concomitant treatments including anticoagulants or antiplatelet drugs. The following data were collected before and 4 days after the first anakinra injection: swollen joint count (SJC), tender joint count (TJC), pain score on a visual analogue scale (VAS, 0-100 mm) and C-reactive protein (CRP) level. A good response was defined according the evaluation of the physician or documentation in the chart of the phrase "good response" after anakinra treatment.

Results: We included 33 patients ( 24 women; mean age $79.2 \pm 12.8$ years). Mean duration of acute arthritis $13.2 \pm 12.9$ days. CPP arthritis was confirmed by the presence of CCP crystals in synovial fluid in $28 / 33(84.8 \%)$ patients. For the remaining 5 patients, the diagnosis was confirmed by CPP deposition features seen on imaging. Corticosteroids, NSAIDs and colchicine were previous treatments, without significant improvement in 12 (36.4\%), 7 (21.2\%) and 18 (54.5\%) patients, respectively. The mean dose of corticosteroids was $20.8 \pm 8.2 \mathrm{mg} / \mathrm{day}$. Among the 33 patients, 32 had a documented visit at day 4 . The number of good responders was $27(81.8 \%)$. At day 4 , patients showed decreased mean VAS pain score (from $64.8 \pm 26.5$ to $21.2 \pm 19.7 \mathrm{~mm}, \mathrm{p}<0.0001$ ), TJC $(5.8 \pm 5.0$ to 1.0 $\pm 1.0, \mathrm{p}<0.0001)$, SJC $(3.9 \pm 2.7$ to $0.9 \pm 1.0, \mathrm{p}<0.0001)$ and CRP level $(116.1$ \pm 71.6 to $26.0 \pm 23.1 \mathrm{mg} / \mathrm{L}, \mathrm{p}<0.0001)$. Anakinra was well tolerated. Only one patient had pneumonitis that was resolved with oral antibacterial agents.

Conclusions: Our results suggest that anakinra could be a relevant alternative for managing acute CPP arthritis, leading to rapid relief of inflammatory symptoms, with a good tolerance.

Disclosure of Interest: None declared

DOI: 10.1136/annrheumdis-2018-eular.4956

\section{FRI0244 LIFE STYLE FACTORS AND COMORBIDITIES IN GOUT PATIENTS COMPARED TO THE GENERAL POPULATION}

M.I. Dehlin ${ }^{1}$, L. Scheepers ${ }^{1}$, A. Landgren ${ }^{1}$, L.T. Jacobsson ${ }^{1}$, U. Lindström ${ }^{1}$, E. Klingberg ${ }^{1}$, U. Bergsten' ${ }^{2}{ }^{1}$ Dept of rheumatology and inflammation research, Institution of Medicine, Sahlgrenska Academy, University of Gothenburg, Gotenburg; ${ }^{2}$ RandD Department, Region Halland, Halmstad, Sweden

Background: Gout is the most common inflammatory arthritis and relatively much is known regarding its pathogenesis. It is clear that life style factors play a significant role in developing and maintaining disease.

Objectives: This study aimed to analyse lifestyle factors in prevalent gout patients by sex compared to age matched controls from the general population. Methods: All patients above 18 years of age with an ICD 10-diagnosis of gout from Jan 2015 through Feb 2017 listed at any of twelve randomly selected primary health care centres or the rheumatology department at Sahlgrenska University hospital in the Western Sweden Health Care Region (WSHCR) were identified. They were sent a questionnaire, regarding demographics, life style factors such as smoking status, alcohol consumption, physical activity, body mass index (BMl categorised into 4 levels in the analyses) and comorbidities such as diabetes and hypertension. All responders aged 18-84 years were matched to five control individuals, without gout, by sex and age. Control individuals were selected from a random sample of 52348 individuals aged 16-84 years who participated in the National Public Health survey in Sweden year 2015 This survey is a national study on health, lifestyle and living conditions. Alcohol consumption was categorised as none and any with/without binge drinking behaviour. Binge drinking was (liberally) defined as consuming more than four (women) or five glasses (men) on any occasion.

Conditional logistic regression models were used to compare cases and controls with regard to lifestyle factors and comorbidities. Multivariate analyses were also performed, including BMI, smoking status, alcohol consumption, and physical activity.

Results: Of the 1589 invited gout patients, 868 responded and $79.7 \%$ were male. Non-responders were more often young men. Mean age was 69.3 (std:10.5) years for men and 71.8 (std: 9.9 ) years for women with gout.

Male gout patients were in multivariate analyses more likely to be overweight (OR 1.67 (95\% Cl: 1.31-2.14)), obese (OR 2.20 (95\% Cl: 1.64-2.94)), have binge drink behaviour (OR 3.32 (95\% Cl: 2.39-4.62)), and had lower levels of physical activity compared to controls (table 1). Current smoking habits did not differ between male gout patients and controls.

Female gout patients were in multivariate analyses more likely to be overweight (OR 1.87 (95\% Cl: 1.05-3.33)), obese (OR 3.62 (95\% Cl: 1.96-6.72)), and have binge drink behaviour (OR 4.28 (95\% Cl: 1.92-9.53)), but not did not differ with regard to current smoking habits or physical activity compared to controls.

In bivariate analyses, comorbidities such as diabetes and hypertension, were sig nificantly more common in gout patients among both sexes.

Abstract FRI0244 - Table 1. Prevalence of lifestyle factors and comorbidities between gout patients and sex-age matched healthy controls.

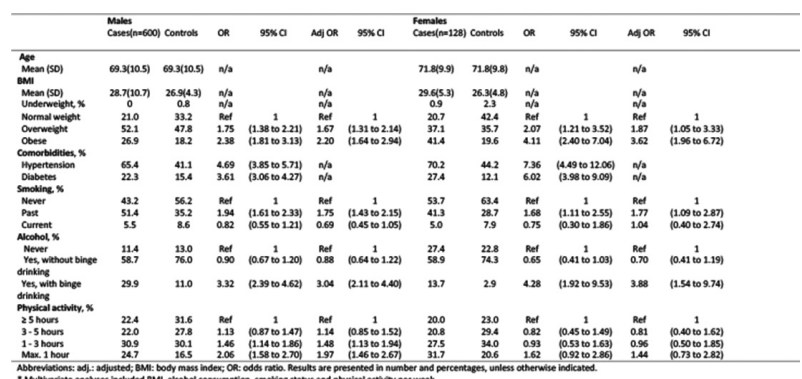

Conclusions: Compared to the general population, patients with gout were more often obese (in particular women) and had higher occurrence of binge drinking behaviour (in particular men). The lower level of physical activity (men) and normal frequency of smoking among gout patients may be a consequence of the high comorbidity rates. 